\title{
The Status of the ARGO Experiment at YBJ
}

G. Aielli ${ }^{\mathrm{a}}$, C. Bacci ${ }^{\mathrm{b}}$, B. Bartolic ${ }^{\mathrm{c}}$ P. Bernardini ${ }^{\mathrm{d}}$, X.J. Bi ${ }^{\mathrm{e}}$, C. Bleve ${ }^{\mathrm{d}}$, S. Bussino $^{\mathrm{b}}$, A.K. Calabrese

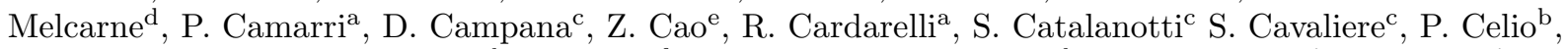
S.Z. Chen ${ }^{\mathrm{e}}$, N. Chenge, P. Creti ${ }^{\mathrm{d}}$, S.W. Cui ${ }^{\mathrm{f}}$, G. Cusumano ${ }^{\mathrm{g}}$, B.Z. Dai ${ }^{\mathrm{h}}$, Danzengluobu ${ }^{\mathrm{i}}$, X.H. Ding ${ }^{\mathrm{i}}$, G. D’Ali Staiti $^{\mathrm{j}}$, B. D’Ettorre Piazzoli ${ }^{\mathrm{c}}$, E. De Marinis ${ }^{\mathrm{b}}$, I. De Mitri ${ }^{\mathrm{d}}$, M. De Vincenzi ${ }^{\mathrm{b}}$, T. Di Girolamo ${ }^{\mathrm{c}}$, G. Di Sciascio ${ }^{\text {, }}$, C.F. Feng ${ }^{\mathrm{k}}$, Zhaoyang Feng ${ }^{\mathrm{e}}$, Zhenyong Feng ${ }^{\mathrm{l}}$, C. Ferrigno ${ }^{\mathrm{j}}$, X.Y. Gao ${ }^{\mathrm{h}}$, Q.B. Gou ${ }^{\mathrm{e}}$, H.H. He ${ }^{*}$, Hongbo Hu ${ }^{\mathrm{e}}$, Haibing Hu ${ }^{\mathrm{i}}$, Q. Huang ${ }^{\mathrm{l}}$, M. Iacovacci ${ }^{\mathrm{c}}$, I. James ${ }^{\mathrm{m}}$, H.Y. Jia ${ }^{\mathrm{l}}$, Labaciren ${ }^{\mathrm{i}}$, J.Y. Li $^{\mathrm{k}}$, H.J. Li ${ }^{\mathrm{i}}$, B. Liberti ${ }^{\mathrm{a}}$, G. Liguori ${ }^{\mathrm{m}}$, C.Q. Liuh ${ }^{\mathrm{h}}$, J. Liuh ${ }^{\mathrm{h}}$, H. Lu ${ }^{\mathrm{e}}$, G. Mancarella ${ }^{\mathrm{d}}$, S.M. Mari ${ }^{\mathrm{b}}$, G. Marsella $^{\mathrm{d}}$, D. Martello ${ }^{\mathrm{d}}$, S. Mastroiannic ${ }^{\mathrm{C}}$, X.R. Meng ${ }^{\mathrm{i}}$, J. Mu ${ }^{\mathrm{h}}$, L. Nicastro ${ }^{\mathrm{g}}$, C.C. Ning ${ }^{\mathrm{i}}$, L. Palummo ${ }^{\mathrm{a}}$, M. Panareo ${ }^{d}$, L. Perrone ${ }^{\mathrm{d}}$, P. Pistilli ${ }^{\mathrm{b}}$, X.B. Qu ${ }^{\mathrm{k}}$, E. Reali $^{\mathrm{a}}$, E. Rossi ${ }^{\mathrm{c}}$, B. Sacco ${ }^{\mathrm{j}}$, L. Saggese $^{\mathrm{c}}$, P.

Salvini $^{\mathrm{m}}$, A. Segreto ${ }^{\mathrm{g}}$, R. Santonico ${ }^{\mathrm{a}}$, P.R. Shen ${ }^{\mathrm{e}}$, X.D. Sheng ${ }^{\mathrm{e}}$, F. Shi $^{\mathrm{e}}$, C. Stanescu ${ }^{\mathrm{b}}$, A. Surdo ${ }^{\mathrm{d}}$, Y.H. Tan $^{\mathrm{e}}$, P. Vallania ${ }^{\mathrm{n}}$, S. Vernetton ${ }^{\mathrm{n}}$, H. Wang ${ }^{\mathrm{e}}$, Y.G. Wang ${ }^{\mathrm{e}}$, C.Y. Wu ${ }^{\mathrm{e}}$, B. Xu ${ }^{\mathrm{l}}$, L. Xue ${ }^{\mathrm{k}}$, H.T Yang ${ }^{\mathrm{h}}$, Q.Y. Yang $^{\text {, X.C. Yang }}{ }^{\text {, G.C. Yu }}{ }^{\text {, A.F Yuan }}{ }^{\mathrm{i}}$, M. Zha ${ }^{\mathrm{e}}$, H.M. Zhang ${ }^{\mathrm{e}}$, L. Zhang ${ }^{\mathrm{h}}$, P. Zhang ${ }^{\mathrm{h}}$, X.Y. Zhang ${ }^{\mathrm{k}}$, Y. Zhang ${ }^{\mathrm{e}}$, Zhaxisangzhu ${ }^{\mathrm{i}}$, X.X. Zhou ${ }^{\mathrm{l}}$, F.R. Zhu ${ }^{\mathrm{e}}$, Q.Q. Zhu ${ }^{\mathrm{e}}$,

(The ARGO-YBJ Collaboration)

aDipartimento di Fisica dell'Università "Tor Vergata" di Roma and INFN Sezione di Roma2 - Italy

bDipartimento di Fisica dell'Università "Roma Tre" di Roma and INFN Sezione di Roma3 - Italy

${ }^{\mathrm{c}}$ Dipartimento di Fisica dell'Università di Napoli and INFN Sezione di Napoli - Italy

${ }^{\text {d} D i p a r t i m e n t o ~ d i ~ F i s i c a ~ d e l l ' U n i v e r s i t a ̀ ~ d i ~ L e c c e ~ a n d ~ I N F N ~ S e z i o n e ~ d i ~ L e c c e ~-~ I t a l y ~}$

${ }^{\mathrm{e}}$ Key Laboratory of Particle Astrophyics, Institute of High Energy Physics, Chinese Academy of Sciences, Beijing 100049, Beijing, China

${ }^{\mathrm{f}}$ Hebei Normal University, Shijiazhuang 050016, Hebei, China

gIASF-INAF, Sezione di Palermo and INFN Sezione di Catania - Italy

hYunnan University, Kunming 650091, Yunnan, China

iTibet University, Lhasa 850000, Tibet, China

jDip. di Fisica e Tecnologie Relative dell'Università di Palermo and INFN Sezione di Catania - Italy

${ }^{k}$ Shandong University, Jinan 250100, Shandong, China

${ }^{1}$ Southwest Jiaotong University, Chengdu 610031, Sichuan, China

mDipartimento di Fisica Nucleare e Teorica dell'Università di Pavia and INFN Sezione di Pavia - Italy

${ }^{\mathrm{n}}$ INAF-IFSI and INFN Sezione di Torino - Italy

${ }^{*}$ Corresponding author. Tel: +86-10-88233167; Fax: +86-

10-88233086; E-mail: hhh@ihep.ac.cn 
The ARGO-YBJ experiment, located at Yangbajing, Tibet, China, performed by a wide Sino-Italian collaboration, is designed to study cosmic rays, sub-TeV gamma ray sources and GeV Gamma Ray Burst (GRB) emission in the northern hemisphere, by means of detecting small size EAS (Extensive Air Shower) using a full coverage RPC (Resistive Plate Chamber) carpet. The central carpet of the detector is installed and put into operation to date, with $1900 \mathrm{~m}^{2}$ of the carpet already operating since December 2004. With a trigger multiplicity of $\geq 60$ hits, corresponding to a primary mode energy of $2 \mathrm{TeV}$, the angular resolution of EAS measurements is $<1^{\circ}$ for showers with more than 500 recorded hits. We report the preliminary results of data taking performed during 2005: the all-sky survey for gamma ray sources and the search for GRBs, as well as the cosmic ray spectrum up to about $100 \mathrm{TeV}$. The Forbush decrease of the cosmic ray flux during January, 2005 is observed using the ARGO data.

\section{The ARGO Detector Installation}

The ARGO apparatus consists of a full coverage array of dimension $74 \times 78 \mathrm{~m}^{2}$ realized with a single layer of RPCs, $280 \times 125 \mathrm{~cm}^{2}$ each. The area surrounding this central carpet, up to $110 \times 100 \mathrm{~m}^{2}$, will be partially instrumented with RPCs (the guard ring). A cluster consists of 12 contiguous RPCs while one RPC is divided into 10 basic detection units (called PADs, each with 8 digital readout strips). A $0.5 \mathrm{~cm}$ thick lead converter will uniformly cover the detector in order to improve the angular resolution.

The central carpet (130 clusters of RPCs) has been installed and put into operation while the guard ring (24 clusters) is currently under construction at the YangBaJing Cosmic Ray Observatory (Tibet, P.R. China, 4300m a.s.l., $\left.606 \mathrm{~g} / \mathrm{cm}^{2}\right)$. The energy threshold of the detector is a few hundreds of $\mathrm{GeV}$ in the full operational mode. With the digital readout ARGO reaches its energy upper limit at about $100 \mathrm{TeV}$, which can be extended to the knee region by means of analog readout(two BigPADs for each $\mathrm{RPC}$ ). GRBs at $\mathrm{GeV}$ region can also be observed by ARGO in scaler mode (SPT: Single Particle Technique). The ARGO detector will be used for a full sky survey, searching for stable and transient gamma ray sources, and for studies on the cosmic ray spectrum etc.

\section{Operation of the ARGO Detector}

The overall detector modularity allowed us to start ARGO data taking using the mounted clusters while mounting and setting-up the remaining ones.

\subsection{Digital Readout}

From November 2003 to December 2004, 16 clusters, covering about $690 \mathrm{~m}^{2}$, had been operated for a final test. Since then, 42 clusters (ARGO-42, 1900m $\mathrm{m}^{2}$ ) have been put into operation and the number of running clusters reached 130 in June, 2006. The data acquisition has been running for more than two years. More than 12 TB of data were acquired and transferred to the disk array at Institute of High Energy Physics (IHEP), Beijing, China. The trigger condition [1] required more than 60 fired hits (LM60) for ARGO-42 with the event rate of about $160 \mathrm{~Hz}$. Currently ARGO is running with LM20 and the event rate is greater than $3 \mathrm{KHz}$. The preliminary data analysis shows that the ARGO carpet detector is working properly. Some physics results based on the ARGO-42 data set were reported at the 29th ICRC (Pune, 2005).

\subsection{Analog Readout}

Analog readout electronics [2] has been installed on 24 clusters covering an area of about $1000 \mathrm{~m}^{2}$. The charge readout of each RPC is performed by means of two $1.40 \times 1.25 \mathrm{~m}^{2}$ BigPads. A stable correlation between the mean number of strips and the mean amplitude of a BigPad is found during the operation for more than one and 
a half years. The event size spectra computed using the analog readout and digital readout data showup to cover a large dynamic range in a consistent way (Fig.1).

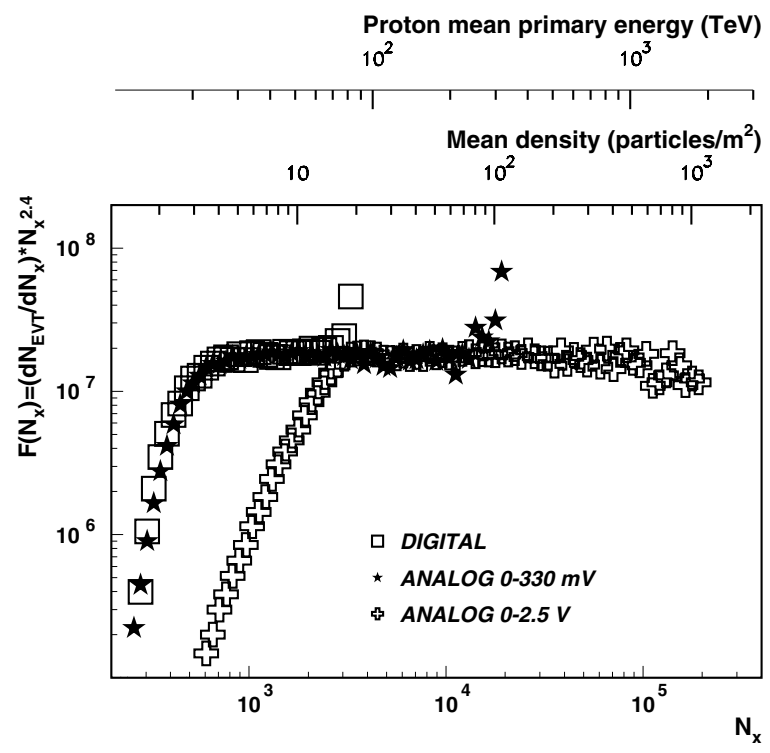

Figure 1. The event size spectra computed using the analog readout and digital readout data.

\section{Calibration of the ARGO Detector}

The performance of the RPCs is measured at YBJ with a telescope realized by $6 \mathrm{RPCs}$ [3]. The resulting efficiency is about $95 \%$, the detector time resolution $<2 n s$ and the average strip multiplicity is about 1.1 strips per hit under a condition of $\mathrm{HV}=7200 \mathrm{~V}$ and a gas mixture of argon (15\%), isobuthane (10\%) and R134a (75\%). These results are consistent with those obtained at sea level [4]. The time calibration is done with two methods, i.e. manual calibration using a standard probe detector and offline calibration $[5,6]$ using selected cosmic ray events with multi- plicity greater than 1000. 240 PADs out of 5040 (ARGO-42) were uniformly sampled among the detectors and calibrated manually. The calibration shows that the relative time difference between PADs is less than 20ns. The difference between off-line calibration and manual calibration follows a Gaussian with a width of $0.4 \mathrm{~ns}$ (Fig.2).

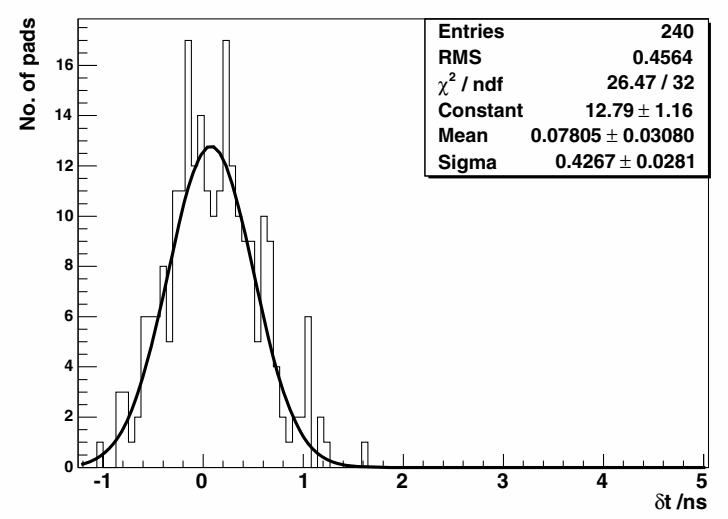

Figure 2. The difference between off-line calibration and manual calibration, superposed with a Gaussian fit.

\section{Performance of the ARGO Detector}

\subsection{Angular Resolution}

The angular resolution has been studied by means of the even/odd method [7]. The experimental results are in very good agreement with MC simulations. The angular resolution strongly depends on the numbers of fired PADs. The opening angle for internally reconstructed protoninduced events is $<1^{\circ}$ when more than 500 PADs are fired.

\subsection{Energy Scale}

A simulation driven by Corsika 6.02 [8] with a detailed detector setup was made in order to find 
the energy scale of the current trigger condition of the ARGO detector [1]. The mode energy of the triggered event distribution is about $2 \mathrm{TeV}$ and the median energy is about $5 \mathrm{TeV}$ for ARGO-42. The threshold is lowered down to a few hundreds of $\mathrm{GeV}$ by setting the trigger condition to LM20.

\subsection{Sensitivities for Gamma Ray Point Source and Gamma Ray Burst Search}

A simulation was carried out to find the sensitivities for both stable gamma ray sources and GRBs. Based on the angular resolution reported above, three algorithms were compared using the average sensitivities from 2000 toy MC experiments. The ARGO experiment is able to detect $\mathrm{TeV}$ gamma ray emission from Crab at a significance level of $18 \sigma$ per year. Taking the standard of $5 \sigma /$ year, the ARGO experiment is expected to observe a point source with a flux of $0.3 I_{\text {crab }}[9]$. The sensitivity curves of the ARGO detector to transient sources [10] are compared with the spectra of EGRET gamma ray bursts GRB930131 and GRB910503 showing that the ARGO detector is able to observe similar events if the spectra extend up to $300 \mathrm{GeV}$ and $700 \mathrm{GeV}$, respectively.

\subsection{Consistency and Operational Stability of the ARGO Detector}

Fig. 3 shows the single rate distribution of the 130 clusters of the central carpet, from which one can see that the variation of the ARGO clusters is about $5 \%$. The efficiency of the RPC detector is stable during the operation since November 2003. The temperature in the experimental hall is monitored and found to be above $5^{\circ} \mathrm{C}$ which guarantees the normal operation of the RPC detector. Gas flow, high voltage, current and single particle rate are monitored and found stable. About 4 volumes of gas are exchanged every day. All the clusters are running under the Detector Control System (DCS) [11]. About $0.5 \%$ of dead strips are found at the installation time. The number of dead strips increases by less than $1 \%$ per year.

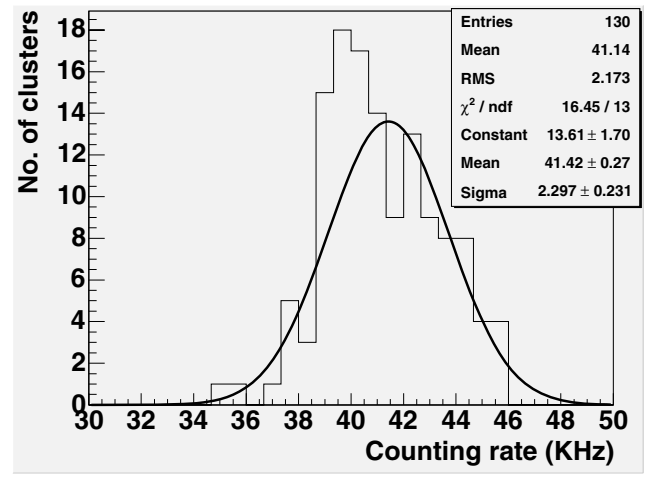

Figure 3. Single rate distribution of the 130 clusters of the central carpet, superposed with a Gaussian fit.

\section{Data Analysis and Physics Results}

\subsection{All Sky Survey for Gamma Ray Sources and GRBs}

The data set used in this analysis has been recorded by ARGO-42 under shower mode from December 24, 2004 to March 23, 2005, for a total running time of 1007 hours. The event rate was $160 \mathrm{~Hz}$. The events with zenith angle $\theta \leq 50^{\circ}$ are considered in this paper. The declination band $-20^{\circ}<\delta<80^{\circ}$, corresponding to $8.3 \mathrm{sr}$ ( $66 \%$ of the celestial sphere) is monitored. No gammahadron discrimination is applied in this preliminary analysis. The analysis of the first data taken by ARGO-42 shows that the detector is working properly. In the 1007 hours of measurement [12] no gamma ray source with an average flux larger than 5 Crab units has been observed in the declination range $20^{\circ}<\delta<40^{\circ}$ (Fig.4). Moreover, significant transient of duration 10-300 seconds has not been detected.

\subsection{Gamma Ray Burst Search using SPT}

The GRB search is done in coincidence with satellite experiments. As a member of the Swift Follow-up project, the ARGO experiment can carry out a complete GRB analysis in "real time". The GRB search started in correspondence with 


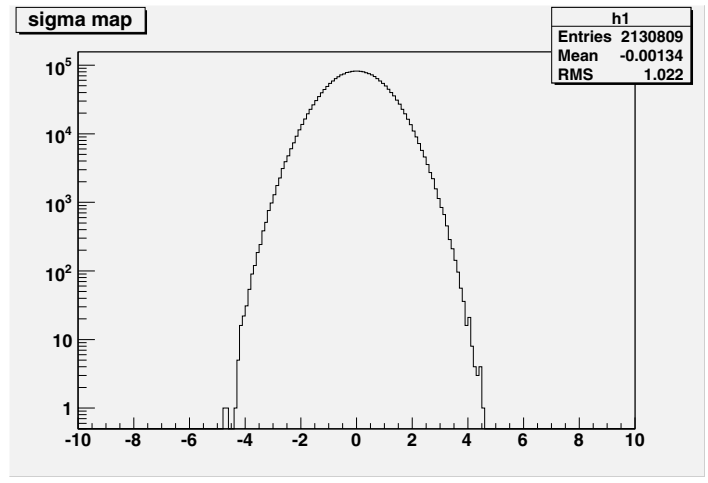

Figure 4. The significance distribution of the bins of the sky map.

the first GRB detected by Swift on December 17, 2004. Up to June 2006, 28 GRBs detected by satellites were in the field of view of this analysis $\left(\theta<40^{\circ}\right)$. Due to the detector installation, calibration and debugging, reliable data are available for only 16 of these GRBs (see Table 1). Three of them have statistical significance of the signal of 3.7, 3.6 and $2.8 \sigma$, that become $2.9,2.8$ and $1.7 \sigma$ taking into account the number of trials. Thus, no GRB has been detected by ARGO SPT up to now. Corresponding $3 \sigma$ fluence upper limits in the 1-100 GeV energy range were estimated using the known low energy time duration and spectral index. For those GRBs whose redshift is known, the upper limit was calculated including a simple $\gamma \gamma$ absorption model, while $\mathrm{z}=0$ is assumed for the others.

\subsection{Cosmic Ray Spectrum below 100TeV}

The strip size spectrum $\left(N_{s}\right)$ has been measured by using the RPC digital readout [15]. Working in digital readout mode, each induction strip serves as a shower particle counter. A preliminary study of the strip size spectrum up to $N_{s}=10^{4}$, which is due to primary cosmic rays with energies up to about $100 \mathrm{TeV}$, shows a fair agreement with the data of direct measurements (intensity and spectral slope), the RUNJOB model being favoured in particular at low
Table 1

List of GRBs in this analysis

\begin{tabular}{lllll}
\hline GRB & $\mathrm{z}$ & $\gamma$ & $n_{\sigma}$ & $\mathrm{UL}$ \\
\hline 041228 & - & 1.56 & -1.3 & $3.3 \cdot 10^{-4}$ \\
050408 & 1.24 & 1.98 & -2.2 & $\mathbf{9 . 6} \cdot \mathbf{1 0}^{-\mathbf{5}}$ \\
$050509 \mathrm{~A}$ & - & 2.1 & 0.29 & $1.6 \cdot 10^{-4}$ \\
050528 & - & 2.3 & -0.012 & $6.5 \cdot 10^{-4}$ \\
050802 & 1.71 & 1.55 & 0.74 & $\mathbf{1 . 0} \cdot \mathbf{1 0}^{-4}$ \\
$051105 \mathrm{~A}$ & - & 1.33 & 0.90 & $1.4 \cdot 10^{-5}$ \\
051114 & - & 1.22 & 2.8 & $1.9 \cdot 10^{-5}$ \\
051227 & - & 1.31 & 0.93 & $2.5 \cdot 10^{-5}$ \\
060105 & - & 1.11 & 3.6 & $5.9 \cdot 10^{-5}$ \\
060111 & - & 1.63 & 0.82 & $2.5 \cdot 10^{-5}$ \\
060115 & 3.53 & 1.76 & -2.2 & $\mathbf{2 . 3} \cdot \mathbf{1 0}^{-4}$ \\
060421 & - & 1.53 & -0.46 & $1.6 \cdot 10^{-4}$ \\
060424 & - & 1.72 & 1.9 & $4.1 \cdot 10^{-5}$ \\
060427 & - & 1.87 & -1.8 & $1.8 \cdot 10^{-4}$ \\
$060510 \mathrm{~A}$ & - & 1.55 & 3.7 & $2.3 \cdot 10^{-4}$ \\
060526 & 3.21 & 1.66 & 0.75 & $\mathbf{1 . 2} \cdot \mathbf{1 0}^{-4}$ \\
\hline
\end{tabular}

z: redshift

$\gamma$ : spectral index

UL: Upper Limit on the fluence $(1-100 \mathrm{GeV})$ in $\mathrm{erg} / \mathrm{cm}^{2}$. The numbers in bold take into account absorption by the EBL

energies $<50 T e V$ (Fig.5).

\subsection{Forbush Decrease in January 2005 and Solar Activities Analysis Using SPT}

After correction for air pressure, the SPT data from 15th to 27th of January, 2005 are used to search for the Forbush Decrease (FD) that is well measured by devices at lower energies such as neutron monitors. The FD around noon on January 17, 2005 is observed by the ARGO detector. The SPT rates with multiplicity $\geq 1$ and $\geq 2$ clearly decrease correspondingly [18]. All detailed structures of the light curves are similar to the results from neutron monitors. The maximum amplitude of the FD is about $-6 \%$, while the SPT rate of multiplicity $\geq 4$ shows almost no signs of decrease (Fig.6).

The solar flare (Class X7) of Jan 20, 2005, that was connected to a GLE and detected by the Milagro scaler system [19], has been also studied but not detected by ARGO [14]. 


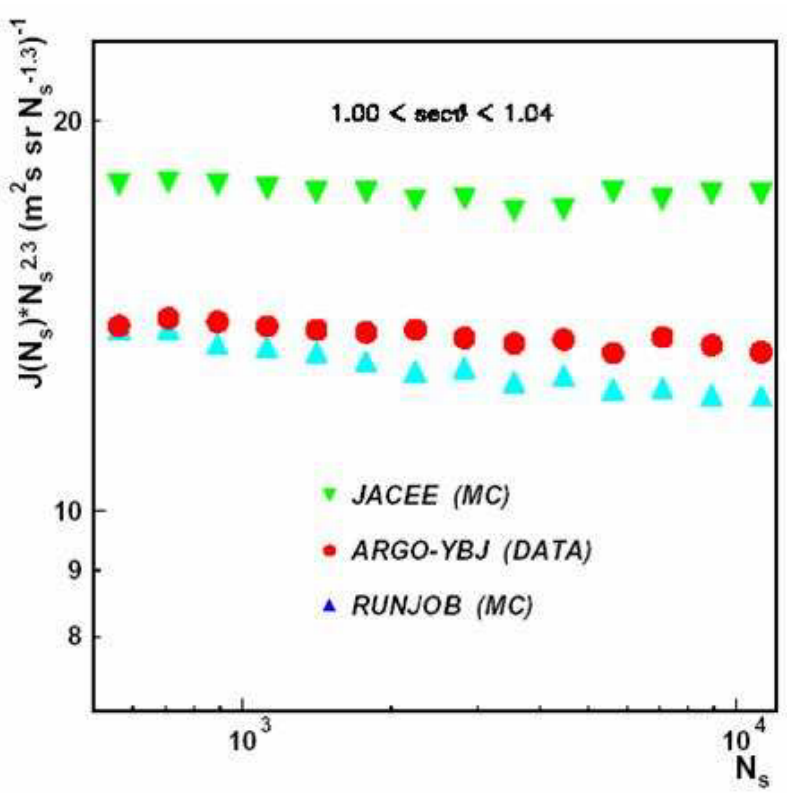

Figure 5. ARGO-YBJ strip size spectrum compared with those expected according to RUNJOB [16] and JACEE [17] composition models of primary cosmic rays.

\section{Looking ahead}

\subsection{Further Installation}

The construction of the central detector carpet of the ARGO experiment has been concluded. In order to complete the whole ARGO detector, the guard ring RPCs will be installed by October of 2006. All installed detectors will be timely merged into the data acquisition system.

\subsection{Calibration of the Analog Readout}

The analog readout of the ARGO RPCs has been calibrated using the digital readout up to 20 particles $/ \mathrm{m}^{2}$. The further calibration will be done by using the Beam Test Facility of the DAFNE accelerator at the INFN Frascati National Laboratories. A parallel calibration is carried out at IHEP, Beijing, by using scintillation detectors that have been calibrated over a dy-
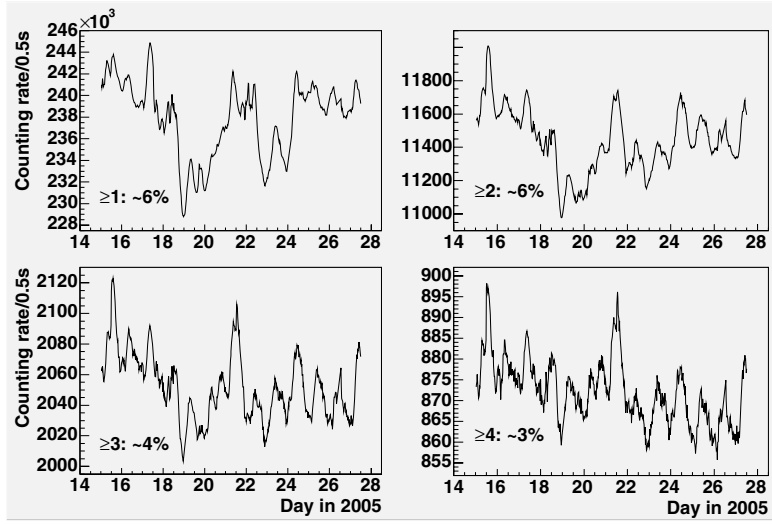

Figure 6 . The Forbush decrease observed with ARGO SPT on Jan. 17, 2005 .

namic range up to $10^{4}$ particles $/ \mathrm{m}^{2}$.

\section{Acknowledgements}

This work is supported in China by NSFC(10120130794), the Chinese Ministry of Science and Technology, the Chinese Academy of Sciences, the Key Laboratory of Particle Astrophysics, CAS, and in Italy by the Istituto Nazionale di Fisica Nucleare (INFN).

\section{REFERENCES}

1. S. Mastroianni et al. for the ARGO Collaboration, 29th ICRC Pune (2005) 5, 311-314.

2. P. Creti et al. for the ARGO Collaboration, 29th ICRC Pune (2005) 8, 97-100.

3. X.D. Sheng et al. for the ARGO Collaboration, 29th ICRC Pune (2005) 5, 151-154.

4. G. Aielli et al. (ARGO Collaboration), Nucl. Instr. Meth., A562 (2006) 92

5. H.H. He et al., 29th ICRC Pune (2005) 5, 143-146.

6. P. Bernardini et al. for the ARGO Collaboration, 29th ICRC Pune (2005) 5, 147-150.

7. G. Di Sciascio et al. for the ARGO Collaboration, 29th ICRC Pune (2005) 6, 33-36. 
8. D. Heck et al., Karlsruhe Report FZKA 6019 (1998).

9. S.W. Cui et al. for the ARGO Collaboration, 29th ICRC Pune (2005) 4, 5-8.

10. X.X. Zhou et al., 29th ICRC Pune (2005) 5, 139-142.

11. P. Camarri et al. for the ARGO Collaboration, 29th ICRC Pune (2005) 8, 89-92.

12. S. Vernetto et al. for the ARGO Collaboration, 29th ICRC Pune (2005) 4, 375-378.

13. J. Greiner 2005 GRB webpage, http://www.mpe.mpg.de/ jcg/grbgen.html.

14. T. Di Girolamo et al. for the ARGO Collaboration, 29th ICRC Pune (2005) 4, 431-434.

15. L. Saggese et al. for the ARGO Collaboration, 29th ICRC Pune (2005) 6, 37-40.

16. V. A. Apanasenko et al, Astr. Phys. 16, 13, (2001).

17. R. Asakimori et al, ApJ. 502, 278, (1998).

18. H.Y. Jia et al. for the ARGO Collaboration, 29th ICRC Pune (2005) 1, 137-140.

19. G. Sinnis, Presentation at the Conference "Towards a Network of Atmospheric Cherenkov Detectors", Palaiseau (France), 27-29 April 2005. 